\title{
LA "RECONVERSIÓN AMBIENTAL" DE LA MINERÍA A CIELO ABIERTO EN ESPAÑA
}

\author{
("ENVIRONMENTAL RECONVERSION" OF THE SPANISH OPENCAST MINING)
}

J. Saiz de Omeñaca y J.A. Saiz de Omeñaca, Laboratorio de Patología Vegetal, E.T.S.I. de Montes-U.P.M.

ESPAÑA

Fecha de recepción: 10-I-96

$113-34$

\author{
RESUMEN \\ La promulgación de normas legales de carácter ambiental ha \\ tenido una influencia considerable sobre la minería a cielo \\ abierto, en especial a causa de las obligaciones de realizar \\ Evaluaciones de Impacto Ambiental y de rehabilitar el \\ territorio afectado por las labores. En este trabajo se discuten \\ los principales aspectos de tal influencia, tanto los positivos \\ como los negativos, con referencias a casos concretos.
}

\section{SUMMARY}

The enactement of Spanish laws concerning the environment has had a considerable influence on the opencast working, particularly due to the obligations of realize environmental impact assessments and the reclamation of mining areas. This paper discusses the major aspects of this influence-both positive and negative- in recent years, making particular references to specific cases.
Palabras clave: Evaluación de Impacto Ambiental, Rehabilitación de explotaciones mineras a cielo abierto, Tecnología del Medio Ambiente.

\section{Introducción}

A lo largo de poco más de un siglo, la minería española ha pasado por situaciones muy dispares. De protagonista de la exportación pasó a sufrir los efectos de la Gran Depresión, fue impulsada por la carrera de armamentos previa a la segunda Guerra Mundial, sometida a colectivización o intervención durante la Guerra Civil y tuvo cierta estabilidad durante los años 40 a 60 con motivo de las nacionalizaciones y de la tendencia a la autarquía de la época. Más recientemente, ha sufrido una intensa reorganización, todavía no fínalizada, a causa de la progresivaapertura de los mercados, de la armonización de la competencia en el seno de la Unión Europea y de la legislación ambiental desarrollada durante la última década.
Key words: Environmental Impact Assessment, Opencast Mine Reclamation, Environmental Technologies.
Si se dejan aparte condicionantes como los producidos por los todavía recientes cambios geopolíticos mundiales, que por alejar los riesgos de un desabastecimiento de recursos minerales desaconsejan el mantenimiento de explotaciones por motivos estratégicos, la causa principal de tal reorganización ha sido, incluso por delante de la presión social, la implantación del "mercado único" europeo. En gran medida, su efecto dinamizador se ha debido a la necesidad de adaptar nuestra legislación al marco legal comunitario, que concretamente en materia ambiental era mucho más completo, complejoy restrictivo; y se ha traducido en una normativa multíplice que en buena medida podría tacharse de laberíntica o'de poco realista.

Con esta normativa se pretende conseguir un cierto control de la contaminación y de otras formas de degradación 
ambiental, así como unas mejores condiciones para la conservación de recursos de la Naturaleza (en sentido postindustrial, es decir, incluyendo aire y estabilidad climática, aguas continentales y oceánicas, morfología y suelos, vegetación y fauna, ecosistemas y paisajes por alguna razón valiosos, etc.); o dicho de otra forma, asignar el mejor uso posible a tales recursos, como bienes que se nos han ido haciendo progresivamente más escasos. Con el actual ordenamiento político-económico y en condiciones ideales, son los mecanismos de mercado los que resuelven "automáticamente" los conflictos de interés que implica la asignación de usos a los recursos escasos, incluido el territorio, pero para que dichos mecanismos actúen adecuadamente es necesario que no existan ni costes ni beneficios externos de importancia, es decir, que cada actividad económica soporte la práctica totalidad de los costes que origina y reciba sin merma apreciable unos ingresos que correspondan a los beneficios que causa (Arrow \& Fisher, 1974; Solow, 1974; Pearce, 1976; Mills, 1978;Burrows, 1980; Daly, 1980; Common, 1988;Martínez Allier, 1989; Montoro, 1990; Larson \& Bromley, 1990; Pearce \& Turner, 1990; Tietemberg, 1990; Azqueta, 1992; Azqueta y Ferreiro, 1994; Bhattacharya et al., 1994, entre otros). Por lo tanto, si se quiere conseguir mediante mecanismos de mercado una mejor gestión de los recursos naturales será necesario conseguir, de una $u$ otra forma, la internalización de tales costes ( $y$ deseablemente también beneficios) externos, de tal forma que repercutan sobre los agentes económicos que los causan y se reflejen en los precios de los bienes y servicios correspondientes, en vez de hacerlo a través del Medio Ambiente sobre otros agentes económicos.

Aparte de la regulación por los mecanismos de mercado, en la práctica, los procedimientos concretos más utilizados en la actualidad (aparte de la mera prohibición de acciones o de la zonificación territorial) consisten en la imposición de cuotas o penalizaciones de cuantía variable, bien según el carácter y el volumen de vertidos o emisiones, bien como contraprestación por los costes de depuración o rehabilitación que la sociedad tenga que afrontar. Dadas las evidentes limitaciones de estos sistemas, resultaría deseable que en el futuro se hicieran más comunes otros, como los basados en la incentivación hacia conductas ambientalmente más deseables mediante subvenciones o exenciones de impuestos, que hoy se utilizan raramente (Saiz de Omeñaca y Saiz de Omeñaca, 1993a y 1993b). En el ámbito concreto de la minería a cielo abierto, además de la legislación que con carácter ambiental afecta a otras actividades que producen importantes modificaciones en el territorio, existen otras regulaciones (un resumen puede verse en Fuentes, 1994), entre las cuales las de mayor trascendencia ambiental son, sin duda, el RD 2994/1982 (BOE 15 nov.82), sobre restauración del espacio natural afectado por actividades extractivas, desarrollado por una Orden del mismo año (BOE 28 nov.84) y el RD 1116/1984(BOE 13 jun.84) sobre restauración del espacio natural afectado por las explotaciones de carbón a cielo abierto y el aprovechamiento racional de estos recursos, desarrollado por otra Orden (BOE 15 jun.84). Estas disposiciones establecen y regulan la obligación de restaurar los espacios afectados por la minería y con otras disposiciones, estatales o autonómicas, llenan el vacío de que adolecía al respecto la Ley de Minas de 1973, que únicamente en su art. 5.3 preveía fijar las condiciones de protección del medio ambiente.

La minimización y corrección de impactos en general y la rehabilitación del territorio en particular no dejan de traducirse en internalización de costes externos, si bien, como es de esperar en los casos de actividades ambientalmente onerosas, pueden suponer un incremento de costes que no sólo amenace la cuenta de resultados, sino que ponga en peligrola supervivencia de las explotaciones. Dado que la minería, en especial la que se realiza a cielo abierto, es una de las actividades humanas que puede producir impactos ambientales de mayor gravedad (AWRA, 1974; Down \& Stokes, 1977; Sendlin et al., 1983; Davies, 1983; Saiz de Omeñaca, 1987; Toy \& Hadley, 1987; Kelly et al. 1988; Ayala et al., 1989; HMIP, 1991; varios autores, 1992, 1993; Riley et al., 1993; Filho, 1994; GIMA, 1994; Macedo, 1994; Paithankar, 1994, etc.), no es de extrañar que los efectos del desarrollo de la legislación ambiental estén siendo lo suficientemente importantes como para hablar de una reconversión ambiental para el sector, ni que en este campo, como en otros, se contemplen con frecuencia las obligaciones ambientales comoobstáculos a la extracción de recursos valiosos para la sociedad, en vez de como mecanismos válidos para conseguir: 1) una toma de decisiones (explotar o no explotar, de una forma o de otra) más acertada; 2) una mejor regulación de los recursos (en el sentido señalado); 3) una armonización de las condiciones de competencia económica; 4) evitar el despilfarro o la degradación, al menos en cierta medida, de los recursos comunes y 5) conservar o conseguir un ambiente que pueda satisfacer nuestras necesidades, sean éstas la obtención de alimentos y materias primas o tengan carácter sanitario o estético.

\section{Algunos factores peculiares}

La minería a cielo abierto produce impactos ambientales considerables, de tipo y grado muy variable, en el lugar de la explotación, en el entorno próximo y aún en lugares muy alejados, siendo en muchas ocasiones de difícil o imposible restauración (véanse, por ejemplo, Griffith, 1980; Bradshaw \& Chadwick, 1980; Banks \& Nickel, 1981; Coppin \& Bradshaw, 1982; RMC, 1987; DEWO, 1989; IBAMA, 1990; IBRAM, 1992; Green, 1992, entre otros y además de bibliografía ya citada). Las características concretas en cada caso dependen en gran medida de la naturaleza del recurso extraído, de las rocas que lo acompañan, de la magnitud de las explotaciones, de los métodos de extracción, del tratamiento posterior y de 
factores locales como el clima, la situación geológica y geográfica, etc., por lo que no cabe una exposición que resuma todos aquellos aspectos. Conviene, en cambio, destacar brevemente, como peculiares de la última década y de nuestro país, cuatro factores de importancia: el crecimiento de la actividad extractiva, la mayor frecuencia de explotación a cielo abierto, la diversidad de nuestro territorio y la calidad ambiental de buena parte del mismo.

El notable incremento de la demanda de minerales y rocas industriales (convencionalmente, no metálicos ni energéticos) a lo largo de los últimos años, en España y en los países de nuestro entorno económico más próximo, ha sido uno de los factores que más ha influido en la generación de impactos ambientales por la minería, pues se ha traducido inevitablemente en un crecimiento de la actividad extractiva, de las plantas de tratamiento, de las instalaciones auxiliares y de la industria asociada. De cara al futuro, resulta previsible que este incremento, mucho mayor que el correspondiente a las menas metálicas, continúe a lo largo de al menos las dos o tres próximas décadas, tanto por el crecimiento del consumo en construcción (áridos, aglomerantes, productos cerámicos, piedra natural, pizarras, etc.) y por la industria química, como por el desarrollo de nuevas aplicaciones.

La mayor frecuencia de la explotación a cielo abierto, sin duda otro factor de importancia, se debe no sólo a la mayor actividad extractiva de minerales y rocas industriales que se acaba de citar, sino también a que diversos condicionantes de índole técnica y económica aconsejan tal forma de explotación para numerosos yacimientos de menas metálicas o de carbones, de tal forma que cada vez es más inexacto relacionar "canteras" (sean formalmente canteras o minas) con recursos minerales de bajo valor unitario. De por sí, esto se traduce, en términos generales, en un aumento de las superficies disturbadas, pero además, en el caso de las explotaciones de minerales metálicos, al laborarse en general con leyes progresivamente más bajas, se incrementan los volúmenes de tierras y rocas que es preciso remover, el de estériles de todo tipo, la energía que se consume, etc., y consiguientemente el riesgo de que se produzcan efectos indeseables sobre espacios más o menos naturales. De no producirse cambios espectaculares en la economía mundial en general o en las disponibilidades energéticas en particular, tampoco parece posible un cambio de tendencia con respecto a este factor.

Por otra parte, aunque la gran diversidad de nuestra litología y geomorfología, flora y fauna, climatología y suelos, usos del territorio y paisaje, etc., no permite fáciles generalizaciones, sí puede afirmarse que precisamente por ella resulta a menudo especialmente difícil tanto la gestión del medio ambiente en general como evitar o minorar los efectos negativos de la minería a cielo abierto en particular. Los materiales geológicos que se explotan y los que constituyen el entorno de las explotaciones, la altitud, las pendientes y las condiciones morfológicas del emplazamiento, las pautas de circulación de aguas superficiales o subterráneas, los rasgos climáticos, los suelos, la cubierta vegetal y por consiguiente la fauna, los usos del suelo y las características del paisaje, etc., resultan ser extraordinariamente variados, por lo que métodos y técnicas válidas en un lugar pueden ser innecesarios, fracasar o aún producir efectos negativos en otros y la experiencia acumulada no puede aplicarse sin más.

La calidad ambiental de gran parte de nuestro territorio es otro factor de importancia. Si se exceptúa a los países nórdicos, que tienen un clima y una densidad de población muy distintos, Españaes la nación de la Unión Europea con mayor superficie dedicada a laconservación de la Naturaleza o escasamente alterada por actividades humanas no tradicionales. Esto puede dar lugar a que explotaciones españolas puedan encontrarse en situación de desventaja en un mercado altamente competitivo a causa de factores medioambientales, pues el grado de alteración del territorio ya existente, o si se quiere su calidad ambiental, puede hacer que los territorios más degradados soporten actividades extractivas ambientalmente muy agresivas sin un deterioro adicional de importancia; una norma igualitaria no sería entonces equitativa y originaría una disarmonía competencial.

\section{Diez años de evaluación de impacto ambiental}

En la actualidad, resulta impensable no ya que pudieran repetirse sin más impactos como los causados por las explotaciones a cielo abierto en las cuencas de los ríos Tinto y Odiel o en la Sierra de Cartagena, sino casos mucho menos llamativos. Sin duda, en buena medida esto es así a causa del desarrollo o de los cambios en la legislación, en particular por la amenaza que supone el art. 347 bis del Código Penal, que establece el Delito Ecológico: la responsabilidad penal recae sobre los directivos u órganos de gobierno(Directores Generales, Administradores, Jefes o Directores de plantas, instalaciones o explotaciones concretas) de las personas jurídicas, pues son ellos quienes pueden controlar de hecho las fuentes de riesgo y quienes tienen capacidad decisoria y de control sobre la empresa o actividad a su cargo. Se supone que el nuevo Código Penal debe suponer una ampliación tanto de los supuestos de infracciones como de las penas correspondientes; por lo pronto, el proyecto existente incluye catorce artículos sobre delito ecológico en vez del único actual.

Por otra parte, los gestores de las explotaciones a cielo abierto son cada vez más conscientes de que los costes ambientales originados por una explotación pueden suponer para la misma costes monetarios, menores ingresos o pérdida de oportunidades financieras. Las vías pueden ser muy variadas: exacciones de tasas, penalizaciones, 
indemnizaciones o sanciones, en cuantías que tienden a ser crecientes; exigencias que como la restauración de los terrenos directamente afectados por la explotación pueden suponer desembolsos considerables; imposibilidad de acceder a ciertas exenciones de impuestos, subvenciones o vías de financiación en condiciones especiales, etc. Por otra parte, la proyección de una imagen ambientalmente depredadora resulta también cada vez más indeseable, pues puede dar lugar, entre otros problemas, a unas difíciles relaciones con la población que convive con la explotación, con sus representantes sociales y con la Administración. En casos extremos, los costes ambientales pueden suponer un verdaderoriesgo para la explotación, en ocasiones lo suficientemente importante como para amenazar a su continuidad, como prueba el caso de la minería de la Sierra de Cartagena, en el que las consideraciones ambientales fueron un factor definitivo de cara a la clausura de las explotaciones (Vilar et al., 1991), pese a los ensayos de revegetación llevados a cabo, en muy difíciles condiciones, ya durante los años 70 (Fernández Gutiérrez, comunicación personal). No en vano, el conjunto de actuaciones previstas para resolver, en la medida de lo posible, el desastre de Portmán se ha valorado en principio en más de 20.000 millones de pesetas.

La reciente entrada en vigor en España del Reglamento comunitario sobre Sistemas de Gestión y Auditorías Ambientales y la aprobación del Proyecto de Ley sobre el Derecho de Acceso a la Información en materia de Medio Ambiente deben significar un mayor control de conductas ambientalmente indeseables $y$, consecuentemente, mayores riesgos para los infractores, al menos si se da una acción ciudadana de mayor envergadura.

\section{Un cambio de mentalidad}

Efectivamente, si los cambios en la legislación llegan a ser realmente trascendentes es porque, al igual que en otras naciones, dichos cambios han venido precedidos, acompañados o al menos seguidos por otros, en particular en el campo de la percepción y de las actitudes sociales (Beale, 1980; González Bernáldez, 1985; Ramos, 1987, 1993; Llano, 1988; Benayas, 1990; Montoro, 1990; Daly, 1990; Nicolaiesen, 1991; Azqueta, 1992; Azquetay Ferreiro, 1994 , etc.). Ciertamente, diversos organismos de la Administración, en particular en ámbitos autonómicos, continúan aceptando ( $\mathrm{y}$ aún realizando) informes sobre Evaluación de Impacto Ambiental cuya finalidad parece ser "demostrar" que "no pasa nada", o no exigen la ejecución de las medidas correctoras correspondientes; admiten Planes de Restauración que como mínimo habría que calificar como muy pobres, o descuidan su ejecución; desatienden la vigilancia y la práctica sancionatoria, o parecen incapaces de hacer efectivas las sanciones. Sin embargo, se percibe que tanto a nivel político como administrativo se tiende a una mejor comprensión de la importancia de los problemas ambientales, se intenta aplicar la legislación de carácter ambiental de una forma más estricta y se deja de ver a la Evaluación de Impacto Ambiental o a los Planes de Restauración como obstáculos a la labor a realizar, para comprender que se trata de instrumentos para conseguir una mejor asignación de uso a ciertos recursos o la degradación de otros.

Este cambio de mentalidad ha sido incluso reconocido judicialmente, con motivo del proceso por el vertido de estériles en la Bahía de Portmán (Cartagena) durante los años 80. El lavadero de mineral "Roberto" arrojó a la bahía un total de 50 millones de toneladas de residuos sólidos, con lo que dio lugar a la contaminación de las aguas marinas con $\mathrm{Cu}$ y otros metales, así como a varios efectos espectaculares: la colmatación de la bahía, el retroceso de centenares de metros de la línea de costa, la desaparición de un puerto pesquero y de un espacio de esparcimiento y la formación de un depósito cuya removilización podría traer nuevas consecuencias negativas para la zona(Faraco, 1986; González y Baños, 1987). La sentencia (1993) consideró inadmisible lo sucedido y admitió la existencia de múltiples consecuencias perniciosas y la gravedad de los daños, pero absolvió a los acusados de delito ecológico, pues estimó que "tal situación siempre ha sido amparada por la Administración, porque en otras épocas han primado los intereses laborales y económicos por encima de los medioambientales".

Las canteras de dolomita de Montehano pueden proporcionar también un buen ejemplo de este cambio de mentalidad. La explotación, que comenzó en 1942, se sitúa en un medio de gran calidad ecológica y paisajística, concretamente en una montaña subcónica prácticamente rodeada por las Marismas de Santoña (Cantabria), que juegan un importante papel en la migración de aves acuáticas, en la alimentación y reproducción de otras especies, en el paso de nutrientes a la plataforma continental, etc. Tras diversas vicisitudes, entre ellas diversos enfrentamientos de asociaciones científicas o ecologistas con la Administración y una condena de la CE al Reino de España (nov. 1990), se creó en las marismas un Refugio Nacional de Aves Acuáticas (Decreto 30/1987 de la Diputación Regional de Cantabria), seles dotóde protección preventiva (Decreto 43/1991 id.) y más tarde fueron declaradas Reserva Natural (Ley 6/1992). A requerimiento de la Administración, el viejo Plan de Restauración de las canteras de Montehano, que contaba con unas pocas páginas y que en forma alguna podía considerarse operativo, fue substituido por otro (Saiz de Omeñaca et al., 1992 y 1994a; Solar et al., 1993), elaborado de acuerdo con el entonces vigente Plan de Labores de las canteras, que preveía la continuidad de la explotación durante 62 años. No obstante, la declaración de Reserva Natural ha llevado a la paralización de las labores y resulta previsible que a más largo plazo se cierren también las actividades industriales asociadas. Aunque el Plan de Restauración no tendrá que realizarse íntegramente, puede representar 
una interesante piedra de toque: el tema ha sido muy controvertido, los impactos causados son notables y los costes de ejecución de la parte del Plan que es preciso realizar son bajos. Si en este caso no se lleva a cabo una minimización de impactos ambientalmente satisfactoria, será difícil creer que se realice en otros que tienen menos factores a su favor.

\section{Rehabilitación de explotaciones a cielo abierto activas}

A lo largo de la última década,llevadas por la nueva situación, muchas empresas han comenzado a realizar importantes labores de rehabilitación en terrenos afectados por la minería a cielo abierto, también por explotaciones previas al RD 2994/1982. Es el caso de las canteras de Cuchía, situadas en la margen derecha de la desembocadura del Saja-Besaya (Cantabria), en la que se obtiene caliza con destino a la industria química desde 1927 y que se encuentra en susúltimas fases por agotamiento de reservas. De acuerdo con el RD citado, la empresa propietaria presentó un estudio de Impacto Ambiental (1983) y más tarde un Proyecto de Restauración (1988). La rehabilitación propiamente dicha del espacio afectado por la explotación, casi $100 \mathrm{Ha}$, comenzó en 1991, se está llevando a cabo por etapas, determinadas por el avance de la explotación, está previsto se extienda durante diez años y pretende obtener un espacio con características de naturalidad, bien integrado en el territorio que le rodea (Maisterra Arroyo, 1990; Mamano, 1993 y comunicación personal). El aprovechamiento de la capacidad natural de regeneración, en especial con respecto al desarrollo de vegetación y de humedales en la plataforma de cantera; la selección de las especies aimplantar, en condiciones con frecuencia difíciles, dando preferencia a las autóctonas; el uso estratégico del escaso suelo disponible y de materiales que pueden substituirlo; y el modelado final, en especial de los antiguos frentes, según criterios paisajísticos, puede conseguir, en relativamente poco tiempo, un espacio de notable calidad ambiental, apto para la vida espontánea y que ya está siendo colonizado (en particular por las aves acuáticas), al menos si la presión urbanística, común al litoral en la región, respeta el lugar. Podrían citarse otros casos, pues no se trata de un ejemplo aislado. Así, la S.A. VascoLeonesa recupera, en León, antiguas escombreras mediante remodelado del terreno (en particular, disminución de pendientes), establecimiento de drenajes, incorporación de humus, plantaciones, etc.

Naturalmente, resulta más ventajosoincluir la restauración en el proyecto minero desde un principio, integrándola con la explotación, pues en general la gestión es más sencilla, los impactos ambientales más reducidos, los costes económicos menores y la rehabilitación más rápida. Es el caso de las minas a cielo abierto de ENDESA en Val de Ariño (Andorra, Teruel), que laborea un yacimiento alargado por transferencia entre paneles, de tal forma que con el estéril de una zona se recubre la explotada con anterioridad; esto permite una ocupación reducida de terrenos exteriores al hueco de explotación, una mejor gestión del suelovegetal y la simultaneidadentre extracción y rehabilitación, pues ésta se lleva a cabo en cada zona subsiguientemente a aquélla. En 1993, tras 11 años de explotación, se daban por recuperadas unas $200 \mathrm{Ha}$ (70 de taludes), parte de las cuales habían entrado en producción (cereal de secano, usos ganaderos, frutales), mientras que otras sostenían masa forestal ovegetación no productiva en términos monetarios (Marrodán y Abril, 1993). En mina Emma (ENCASUR, Puertollano, Ciudad Real) se laborea de forma similar, destinándose la mayor parte de los terrenos que se dan por restaurados al cultivo de cereales, $\mathrm{y}$ en menor medida a pastizales o a sostener vegetación arbóreo-arbustiva propia de la zona (Lorenzo y Apodaca, 1994).

\section{Rehabilitación de explotaciones abandonadas}

La rehabilitación de explotaciones a cielo abierto abandonadas antes de la promulgación de la normativa legal actual, que suele considerarse como un problema de más difícil solución a causa de factores como la escasez de datos y la falta de una entidad responsable de los trabajos, ha comenzadoa ser abordado también, tanto por la iniciativa privada como por la Administración. En el primer caso, la razón suele ser la revalorización del terreno, sea por su proximidad a áreas urbanas (como en el Gran Bilbao) o por la posible utilización del territorio con fines de desarrollo turístico/urbanístico (como se pretende en Portmán); la rehabilitación puede ser también justificable en términos monetarios si los huecos de explotación se utilizan con fines industriales o para establecer vertederos de sólidọs en condiciones controladas (por ejemplo, Azurmendi et al., 1990; Saiz de Omeñaca, 1991; Saiz de Omeñaca et al., 1993a, 1994d). En el segundo caso, las acciones se suelen justificar por su interés social: eliminar riesgos para personas o propiedades, suprimir una fuente de contaminación (frecuentemente vía aguas subterráneas), mejorar la estética de un paisaje, conservar ciertos recursos (agua, suelos, fauna o flora espontáneas), conseguir suelo para usos públicos, etc.

Sin duda, uno de los planes de rehabilitación de zonas mineras más ambicioso en España es el puesto en marcha por la Junta de Andalucía para la recuperación de las cuencas piríticas de los ríos Tinto y Odiel (JA, 1993a y 1993b; Serrano, 1994; Viñas Bosquet y López Fernández, 1994)), cofinanciado al $60 \%$ con fondos FEDER por la UE. Los objetivos del Plan pueden sintetizarse en: 1) eliminar la contaminación por aportes ácidos procedentes de la oxidación espontánea de minerales sulfurosos en explotaciones abandonadas, para evitar que estos aportes, fundamentalmente vía aguas, degraden (como han degradado a través de la historia) corrientes fluviales, contaminen la ría de Huelva, etc., por medio del taponamiento de desagües de efluentes, la reducción de 
entradas de agua a las explotaciones, la retirada de residuos o cobertura de los mismos con arcillas, en algunos casos la neutralización de efluentes, etc.; 2) minorar los impactos paisajísticos causados por las excavaciones, taludes, escombreras, balsas, contrastes cromáticos y demás, mediante la corrección de las alteraciones morfológicas, la restauración de balsas y canales, la implantación de pastizales y otra vegetación, etc. 3) rehabilitar espacios improductivos, preparándoles para actividades de carácter forestal o social, en particular mediante la reforestación de superficies abandonadas; 4) descontaminar o enmendar suelos; y 5) evitar riesgos, particularmente de erosióny relacionados con la misma, mediante controles de drenaje y revegetación.

Otros buenos ejemplos pueden ser la recuperación de antiguas graveras en el tramobajo del río Jarama (Madrid), en la que las necesidades de conservación de recursos hídricos ha hecho preciso renunciar al establecimiento de lagunas (M.J. Fernández Casals, comunicación personal), pese a que suelen considerarse elementos principales cuando se planifica la rehabilitación de este tipode explotaciones (por ejemplo, Andrews \& Kinsman, 1990; Brookes, 1992); el establecimiento, en terrenos degradados por la minería a cielo abierto cercanos a la margen izquierda de la ría de Bilbao, lugar densamente poblado y con escasas dotaciones para esparcimiento y recreo, del gran parque público de La Arboleda (Palacio, 1991), en el que se ha optado por compaginar actividades de recreo (con o sin instalaciones) con recuperación, por conformar hábitats para la fauna espontánea mediante la reintroducción deflora autóctona y por conservar parcialmente el paisaje minero, por supuesto, controlando riesgos y aprovechando elementos, como es el caso de las masas de agua (observatorio de aves, acondicionamiento para remo o paseo) o de las zonas deprimidas (ajardinamientos e instalaciones); o diversos casos de rehabilitación de canteras abandonadas en Álava, en los que se ha compaginado el vertido de inertes y la recuperación (Gómez Chico, comunicación personal). No faltan tampoco casos en los que, más que una rehabilitación, se ha adecuado un espacio, incluso sin considerar suficientemente su uso anterior (Saiz de Omeñaca et al., 1993b).

Posiblemente, el caso de rehabilitación de unas antiguas minas a cielo abierto más discutido sea el de CabárcenoObregón (Cantabria). El yacimiento se formó al fosilizarse un antiguo karst de torres con un recubrimiento de arcillas, limos, arenas y óxidos férricos ( Gómez de Cos et al., 1980; Saiz de Omeñaca et al., 1981; Rohou et al., 1986; Flor y Martínez, 1989; Gil et al., 1990). Este recubrimiento es el que se explotó, desde tiempo inmemorial (al parecer se obtuvieron ocres ya en época prerromana y en la Historia Natural de Plinio el Viejo se cita un gran yacimiento en la zona), mediante métodos más o menos artesanales, por lo que quedaron progresivamente al descubierto gran parte de las impresionantes formas del karst. Laadecuación del lugar con fines de esparcimiento, en realidad una idea tan afortunadacomo antigua, ha supuesto una rehabilitación de hecho del lugar, muy discutida tanto económica como ambientalmente, entre otros por aspectos como el tipo de parque que se ha buscado, falto de naturalidad y de estabilidad, y por los elevadísmos costes de construcción y de mantenimiento.

No está de más señalar que en el caso de explotaciones antiguas puede ser complicado precisar cuáles han de ser los objetivos de la rehabilitación, no sólo por tener que dar solución a disyuntivas como elegir entre una restauración lo más completa posible o buscar un espacio de otra forma más "útil", sino también ante la duda de si deben preservarse explotaciones que pueden tener valor histórico. Algunos casos, como el de las minas auríferas de Las Médulas (León), explotadas por los romanos durante los siglos II a IV, que han dado lugar a un paisaje extraordinario, que son todavía hoy objeto de arqueología y que han sido declaradas Monumento Nacional, ofrecen pocas dudas; sin embargo, la decisión es con frecuencia más difícil, como sucede en la Sierra de Cartagena, donde se superponen labores fenicias, romanas, árabes, medievales, modernas y contemporáneas.

\section{Perspectiva}

\section{Un mercado forzosamente local}

En princip1o, como cualquier otra actividad económica, nuestras explotaciones a cielo abierto deberán estar a la altura de las de los demás países de la Unión Europea y ser capaces de competir con ellas. Esto, que parece un reto de importancia, entre otras razones porque nuestras empresas están teniendo queafrontar la "reconversión ambiental" con posterioridad y a lo largo de un período de tiempo más reducido que las de la antigua $\mathrm{CEE}$, no lo es tanto en el caso de los recursos minerales de bajo valor unitario, entre ellos la gran mayoría de los destinados a ser materiales de construcción. De hecho se trata de una competencia muy restringida, pues en general tales recursos carecen de un auténtico valor de escasez y con frecuencia unas fuentes pueden ser substituidas por otras, lo que se traduce en que el precio del producto no resulta competitivo si los costes de transporte son elevados. Por ello, resulta forzoso acudir a fuentes locales, y cuando no existen otienen una producción insuficiente, como sucede con frecuencia en el caso de las grandes obras públicas, para no incurrir en costes desproporcionados se abre una nueva cantera.

En su dimensión medioambiental, la gestión de nuestras explotaciones, además de considerar las variables económicas, técnicas, etc., tradicionales deberán acoger las trasformaciones necesarias para ser "ecológicamente limpias", o utilizando una expresión más adecuada, ambientalmente responsables. A este respecto, la peculiaridad que se acaba de señalar es también de prime- 
ra importancia, pues no sólojustifica la apertura de nuevas canteras para satisfacer cualquier demanda local de importancia, aunque su vida se prevea muy corta, sino que en combinación con la litología y otros factores locales predetermina en cierta medida la zona en la que han de abrirse; de esta manera, la necesidad de preservar un paraje por alguna razón valioso puede enfrentarse a incrementos significativos de costes, en particular a causa del transporte, y encontrar las lógicas resistencias. Por otra parte, tal peculiaridad, es también la que está en el fondo de la inconstancia oportunista de muchas explotaciones y en la existencia de numerosas irregularidades administrativas, incluidas la falta de depósitos o avales que garanticen la ejecución de labores de restauración y la apertura de canteras "piratas", situaciones que con frecuencia se relacionan con importantes costes ambientales.

\section{El "ambiente" legaly lasrelaciones con la Administración}

Si se considera la pluralidad de normas españolas y comunitarias con carácter ambiental (se calcula que un tercio de la normativa comunitaria tiene tal carácter y que las normas con trascendencia ambiental en España son más de 4.000), la inexiistencia en España de algo parecido a una Ley de Medio Ambiente que sirva de núcleo legal básico (el art. 45 de la Constitución es insuficiente en este sentido)y que son muchos los organismos con competencias medioambientales, parece que aún cabe esperar la existencia de cambios en el marco legal y, tal vez con mayor razón, en la forma de aplicarlo. Concretamente, sería de desear que la Administración, además de promulgar disposiciones legales, se ocupara de que fueran claras, realistas y operativas, que obligaran realmente a minorar impactos ambientales y a recuperar en la medida de lo posible; así como de procurar su cumplimiento con seriedad y eficacia, aunque entren en conflicto con intereses industriales, urbanísticos o turísticos. Para ello, sería sin duda de utilidad que todas las Comunidades Autónomas dispusieran de un plan de actuación, basado en una estimación realista de las necesidades futuras, en información suficiente sobre calidad ambiental del territorio que gestionan y en un censo y clasificación de todas las explotaciones, estén activas o abandonadas, tengan sus papeles en regla o estén en situación “irregular", etc., según sus principales características reales y actualizadas (propiedad, situación geográfica y geológica, características del entorno que pudiera ser afectado, volumen de reservas, recurso explotado, técnicas y tratamientos utilizados, producto obtenido, etc.). Esto facilitaría el ordenamiento del sector, el control de impactos y el seguimiento de las restauraciones hasta su cumplimiento; asimismo, permitiría informar a explotadores, propietarios del terreno y ayuntamientos de sus respectivos derechos y deberes, facilitando su coordinación.

Por otra parte, cabe señalar que la "reconversión ambiental" puede resultar un reto para muchas explotaciones medianas o pequeñas, en especial en el caso de aquéllas de actividad inconstante y siempre que el equipo técnico fuera reducido o tuviera una mentalidad de otros tiempos. Cierto que en estos casos se debe acudir a la consultoría ambiental, pero resultaría más realista que la Administración facilitara modelos de actuación para evitar ciertos impactos (en particular aquéllos que siendo poco aparentes pueden, sin embargo, revestir gravedad), para asesorar respecto a aspectos especialmente conflictivos (como es el caso del elemento fauna,'uno de los más difíciles de valorar y de los que más problemas ocasiona a los gestores de las explotaciones, Jaramillo, 1994) o para conseguir una rehabilitación efectiva a bajo coste, especialmente en aquellos casos que por su frecuencia y características fueran típicos de una región. No está de más tampoco señalar la especial dificultad que suponen las numerosas explotaciones en situación administrativa "irregular", pues no sólo implican competencia desleal y graves problemas de control, sino que su clausura sin más puede suponer importantes problemas ambientales, incluso muy superiores a los que puedan derivarse de su pervivencia(en especial cuando con motivo del cierre no se lleva a cabo labor de restauración de ninguna clase, se abandonan maquinaria, instalaciones y efectos sin más, etc.).

También puede ser delicado el caso de muchas explotaciones a cielo abierto antiguas, con grandes extensiones sin restaurar, tal vez en situación económica delicada, incluso con importantes pérdidas acumuladas, en especial si proporcionan numerosos puestos de trabajo. En estos casos es comprensible la resistencia a ejecutar una buena labor de rehabilitación y parece justificable la concesión de ayudas por parte de la Administración; pero en cambio, parece escasamente lógico que tales ayudas se destinen a subvencionar, tal vez de forma encubierta, la continuidad de una explotación que ya no tiene razón de ser, que dista de ser rentable o que produce elevados costes ambientales que más adelante se traducirán en otros económicos, aunque sea de forma indirecta o intangible, y que tal vez supongan elevados costes de rehabilitación. En casos como estos, la Administración debería estudiar soluciones, pero no para subvencionar la continuidad de pérdidas y la generación de mayores problemas ambientales, sino encaminadas a conseguir una auténtica rehabilitación, bien integrada en el entorno, que no suponga riesgos de ningún tipo y que busque un uso posterior idóneo, etc.: se obtendría un mejor rendimiento de los fondos utilizados y se evitarían costes posteriores, sin olvidar que las labores de restauración también precisan mano de obra. "Convertir a los mineros en jardineros" sería más que una frase.

Sin duda, en muchos casos, será probablemente necesario llegar a un compromisoentre explotación y conservación. Por ejemplo, la obtención de áridos de machaqueo en gran parte del norte de España se realiza a partir de la explotación de rocas carbonatadas, en las que por carstificación abundan las cavidades de diverso tipo, en las que son frecuentes 
la existencia de fauna hipógea, tal vez única en el mundo, los depósitos arqueológicos e incluso las pinturas, etc. (Saiz de Omeñaca et al., 1990). El problema se complica en este caso porque muchas de esas cavidades no han sido estudiadas, o lo han sido sólo parcialmente, o incluso no se conoce su existencia; tan sólo puede asegurarse que en una formación geológica determinada puede haberlas y tal vez ni siquiera sea posible señalar, con un mínimode garantías, no ya su posible valor sino incluso la probabilidad de que existan. Sin duda, la gran variedad de situaciones debiera en buena lógica traducirse en desenlaces diferentes y sería absurdo pretender que por precaución siempre en beneficio de la conservación. Algo parecido podría argumentarse en muchos parajes españoles en relación con el paisaje, con lugares de interés arqueológico o histórico y con otros recursos no valorados en términos monetarios o en general mal regulados por los mecanismos de mercado.

\section{Objetivo: reducir el "input medio ambiente"}

De acuerdo con la normativa desarrollada, el control de la degradación ambiental se realiza, bien fijando unos máximos paradeterminadas substancias en vertidos oemisiones, bien mediante la exigencia de unos mínimos de calidad para parámetros físicos, químicos o biológicos expresivos de la misma. Esta segunda vía suele dar lugar a mayores dificultades de control, pero resulta más adecuada para evitar la degradación de sistemas especialmente frágiles, para armonizar emisiones, vertidos o actuaciones con la capacidad de absorción de territorios determinados y para valorar los efectos reales cuando los agentes contaminantes o degradantes intervienen en procesos complejos o tienen carácter sinérgico. En el control ambiental de las explotaciones a cielo abierto se utilizan ambas vías, por ejemplo, en el primer caso, nivel de ruidos producido o calidad de las aguas que se vierten; y en el segundo, diafanidad o $\mathrm{pH}$ de las aguas de corrientes afectadas o porcentaje de suelo cubierto por vegetación. No obstante, el objetivo no es conseguir unos números favorables, sino "consumir" menos medio ambiente.

Desde un punto de visto práctico, la idoneidad de la ubicación de las nuevas canteras, compaginando criterios monetarios y ambientales, debiera ser la primera meta a conseguir: tan absurdo resulta no considerar que la explotación en un paraje determinado puede ser ambientalmente indeseable, como admitir incrementos de costes significativos por forzar una ubicación en un lugar de difícil acceso, o en general a causa de mayores costes de transporte, sin un motivo realmente grave. La diversidad de nuestro territorio hace difícil mayores generalizaciones.

Una gestión consciente de los impactos ambientales que puede causar la minería a cielo abierto y decidida a evitarlos, es en general capaz de reducirlos a niveles comparativamente bajos. La problemática puede ser muy variada, pero al menos puede señalarse que un control de riesgos o una rehabilitación no son en forma alguna aceptables si como mínimo no eliminan las fuentes de riesgos y no permiten obtener, una vez finalizada la explotación, un terreno estable y capaz de sostener algún uso. No está de más aclarar que los riesgos que se mencionan no son sólo los relacionados con la morfología en la zona explotada (desprendimientos, hundimientos, etc.), sino todos los no irrelevantes debidos a la actividad, incluso los que puedan producirse a largas distancias, tras largo tiempo o a través de procesos geológicos que parezcan diluir posibles responsabilidades; que al hablar de estabilidad nos referimos a largo plazo (cuarenta, cien años) y sin necesidad de actuaciones humanas posteriores, al menos de importancia y no requeridos por el nuevo uso del terreno; y que determinar éste entre los que sean posibles implica respetar la "vocación" del territorio y considerar tanto los usos aparentemente con más futuro como los tradicionales de la zona.

En la mayor parte de los casos, rehabilitación implica también existencia de un suelo estable, que puede ser pobre pero debe tener capacidad suficiente para sostener una vegetación apropiada, de tal forma que el paisaje pueda volver a ser armónico y atractivo. Por desgracia, con demasiada frecuencia, la "restauración" no pasa de un "maquillaje" obsesivao únicamente centrado en el desarrollo de vegetación, de cualquier vegetación, que se queda en la mera superficie y olvida los efectos a largo plazo o a distancia. Una auténtica rehabilitación (Bradshaw \& Chadwick, 1980; Banks \& Nickel, 1981; Coppin \& Bradshaw, 1982; Sendlin et al., 1983; RMC, 1987; Toy \& Hadley, 1987; DEWO, 1989; Azurmendi et al., 1990; Andrews \& Kinsman, 1990; Ayala et al., 1989; IBAMA, 1990; Green, 1992; varios autores, 1992, 1993; Saiz de Omeñaca etal., 1993a; Riley et al.,1993; Paithankar, 1994; GIMA, 1994, etc.) no puede dejar de: 1) Estudiar las necesidades locales auténticas: tierras para usos agrícolas, para pastoreoo producción forestal; protección de hábitats, parajes, formas, yacimientos arqueológicos o paleontológicos valiosos por motivos científicos, educativos, estéticos o de otro orden; demandas de suelo (urbano o industrial) o de puntos de vertido, espacios para el ocio, etc.; 2) Reconocer la importancia de la estructura interna del terreno, de las aguas superficiales y subterráneas como vehículo de contaminación (sin olvidar la carga sólida), de la hidrología subterránea en relación con la superficial y de otros rasgos o factores capaces de causar daños de importancia, y actuar en consecuencia; 3) Conseguir una auténtica integración ecológicay paisajística en el entorno, mediante el remodelado morfológico, la utilización de especies adecuàdas, la búsqueda del equilibrio de masas, colores y texturas,... En su caso, el establecimiento de una vegetación estable no es tampoco un fin en sí mismo, sino que al traducirse en una mayor capacidad para la fauna, en un mejor control de riesgos, de la contaminación y de los recursos de agua y en general en una mayor calidad ecológicay paisajística, debe servir al objetivoantes señalado 


\section{Objetivo: contener los costes}

En particular, con respecto a explotaciones a cielo abierto, uno de los principales retos a que han de enfrentarse en los próximos años será evitar o mitigar los efectos ambientales indeseables sin que el incremento de costes haga muy onerosa, o inviable en el caso de la iniciativa privada, la operación. Esta meta puede no ser siempre fácil de conseguir, pero tampoco es imposible, en especial en el caso de nuevas explotaciones, en las que la planificación de la restauración se hace previamente a la explotación e integrada con ella, con lo que pueden conseguirse importantes ventajas: se coordinan explotación y restauración, se evita la acumulación de costes al final del proceso, se prevén acúmulos de materiales (suelo, estériles, materiales de baja permeabilidad, oútiles como enmiendas,...) para la restauración, se evitan costosos movimientos de tierras, etc. No en vano, los programas de acción en materia de Medio Ambiente de la CEE y en particular la directiva sobre Evaluación de las incidencias de los proyectos públicos y privados sobre el Medio Ambiente (CEE 85/337), de los que en buena medida deriva nuestra nueva legislación ambiental parten del principio de que la mejor política ambiental consiste en evitar en origen contaminaciones y perturbaciones, enf vez de corregir sus efectos "a posteriori", aunque esto suponga mayores inversiones a corto plazo y quizá un nivel de riesgo financiero más elevado. Efectivamente, la corrección de disfunciones se traduce meramente en simples agregaciones de costes, mientras que si se actúa substituyendo o modificando los procesos productivos se pueden conseguir economías al mejorar la efíciencia en el uso de la energía y demás recursos, depurar los procesos y disminuir los costes correspondientes, aprovechar subproductos y ampliar posibilidades comerciales, maximizar la productividad y minimizar costes monetarios (Saiz de Omeñaca y Saiz de Omeñaca, 1993a y 1993b).

La adaptación del diseño a las condiciones locales no resulta deseable sólo por motivos de integración ecológica y paisajística, sino también porque puede traducirse en unos costes más reducidos. Por ejemplo, la elección de especies vegetales autóctonas o naturalizadas en el lugar contribuye a evitar posibles errores cromáticos o de textura y a proporcionar las condiciones necesarias para la fauna, pero también debe contribuir el éxito de las plantaciones, al tratarse de especies adaptadas a las condiciones locales. A este fin, el uso de planta micorrizada ha mostrado ser de gran utilidad, especialmente en condiciones difíciles (por ejemplo, Ruehle, 1980; Oria de Rueda, 1987; Pirazzi y Gregorio, 1987; Jasper et al., 1988; Rodríguez Barreal, 1989, 1994; Kropp \& Langlois, 1990; Marx, 1991; Saiz de Omeñaca et al., 1994b y 1994c).

La utilización de residuos, procedentes de la explotación o del exterior, es uno de los medios más útiles para la reducción de costes. Puede tratarse de residuos o estériles "inertes" para modelado y relleno de cavidades; de materiales limosos o arcillosos para impermeabilizar; de bloques y cascajos para resolver problemas de estabilidad o para drenaje; de carbonatos, sulfatos, sales de hierro, etc., para evitar impactos "químicos"; de residuos orgánicos (en particular de "compost" de basuras urbanas) o lodo de depuradoras para mejorar el contenido en materia orgánica de los suelos; de aguas residuales para riegos, de cenizas u otros residuos industriales como enmiendas, etc. (por ejemplo, Murray, 1982; Smith, 1988; Mitchell, 1990; Stewart et al., 1990; Solar et al., 1993; Atkinson \& Mitchell, 1994; Saiz de Omeñaca etal., 1994 d; Mandavgane et al., 1994; Bhole, 1994; Maiti \& Banerjee, 1994). Dado que en general desechar estos materiales constituye un problema, incluso es posible que una rehabilitación bien planteada arroje beneficios en sí misma, es decir, sin contabilizar el posible valor monetario del terreno recuperado (Saiz de Omeñaca et al., 1993a). Un especial cuidado en el diseño de bermas, taludes, balsas de sedimentación y escombreras, la prevención de la erosión y de otros riesgos, la utilización de técnicas blandas, el uso de plásticos y otros materiales para disminuir costes en relación con el agua, el empleo inteligente de las posibilidades que ofrecen la regeneración "natural" asistida, las plantas mejorantes o el ganado, el control de los animales salvajes y sin duda también la formación de técnicos y mano de obra especializada, pueden ser otros medios para disminuir costes de rehabilitación en un próximo futuro.

\section{Algunos desarrollos estratégicos}

Resultaprevisible que algunos desarrollos recientes puedan tener en un próximo futuro especial trascendencia, como es el caso de los estudios sobre el polvo, uno de los agentes impactantes más frecuente en el caso de las explotaciones mineras a cielo abierto. En los últimos años, se han realizado importantes avances en la caracterización de partículas con tal procedencia, en particular con respecto a morfología y microquímica, que se suman a los conocimientos acumulados sobre control de polvo, transporte eólico y mineralogía de rocas y sedimentos. Resulta previsible que se realicen esfuerzos complementarios tanto para complementar aquellos estudios como para profundizar en la caracterización de las propiedades físicas de las partículas, en la estandarizarización de la toma de muestras y en el conocimiento de su toxicología (por ejemplo, Barale et al., 1990, 1991a, 1991by 1994; King et al., 1991; Dean et al., 1992; Merefield et al., 1992, 1994, 1995a y 1995b; Scarpato, 1993; Librando, 1995).

También resulta previsible el interés de los tratamientos para suelos contaminados basados en las posibilidades de diversos minerales industriales (zeolitas, vermiculita, tierra de Fuller, kieselguhr, etc.) como enmiendas o adiciones capaces de controlar cationes pesados, acidez y otros 
problemas (Leppert, 1989; Atkinson et al., 1990; Mitchell, 1990; Mitchell \& Atkinson, 1991; Barber, 1993; Bardos, 1993; Smith, 1993; Atkinson \& Mitchel, 1994). La utilización de biosensores para estimar estreses impuestos por la contaminación, la investigación de propiedades sobresalientes de diversos organismos (aptitud para la retención de contaminantes, posibilidad de desarrollo en condiciones adversas determinadas, capacidad para mejorar o mantener suelos), la ampliación de la oferta de especies vegetales para rehabilitación, el desarrollo de nuevos materiales (tal vez geotextiles de mayor duración y a más bajo precio) y sobre todo, una profundización en el conocimiento de las características y posibilidades del medio, pueden ser otros desarrollos de especial interés.

\section{BIBLIOGRAFÍA}

ANDREWS, J. \& KINSMAN, D. (1990). Gravel pit restoration for wildlife. A practical manual. R. Soc. for the Protection of Birds.

ARROW, K.J. \& FISHER, A.C. (1974). Environmental preservation, uncertainty and irreversibility. Quarterly Jour. of Economics, 88, 312-319.

ATKINSON, K.; EDWARDS, R.P., MITCHELL, P.B. \& WALTER, C.P. (1990). Roles of industrial minerals in reducing the impact of metalliferous waste in Cornwall. Trans IMM, 99, A158-A 172.

ATKINSON, K. \& MITCHELL, P.B. (1994). The environmental impact of mining and some novel methods of reclamation. In Paithankar, A.G. (ed.), The Impact of Mining onthe Environment: Problems and Solutions, Balkema and Oxford \& IBH, 305-310.

AWRA (1974). Water Resources Problems Related to Mining. Amer. Water Resources Assoc., proc. $\mathbf{n}^{\circ} 18$.

AYALACARCEDO, F.J; VADILLO FERNÁNDEZ, L.; LÓPEZ JIMENO, C.; ARAMBÚRU MAQUA, M.P.; ESCRIBANO BOMBÍN, M.; ESCRIBANOBOMBÍN, R.; FRUTOS GÓMEZ, M. DE; MANGLANO ALONSO, S.; MATAIX GONZÁLEZ, C. Y TOLEDO SANTOS, J.M. (1989). Manual de restauraciôn de terrenos y evaluación de impactos ambientales en minería. Instituto Tecnológico Geominero de España, Serie Ingeniería Geoambiental.

AZQUETA, D. (1992). Social Project Appraisal and Environmental Impact Assessment; A necesary but complicated theoretical bridge. Development Policy Review, 3, 255-270.

AZQUETA, D. y FERREIRO, A. (1994). Análisis económico y gestión de recursos naturales. Alianza.

AZURMENDI, I. ATXABAL, K.; EREÑO, I. y SAIZ DE OMEÑACA, J.;(1990). Rehabilitación de la circulación hidrica en las antiguas explotaciones mineras de Malaespera (Bilbao, Vizcaya). IV R. Nac. de Geol. Ambiental y Ordenación del Territorio, Com., 33-38.

BANKS, P. \& NICKEL, R. (1981). Reclamation and Pollution Control: Planning Guide for small Sand and Gravel Mines. Bureau of Mines, U.S. Dept. of the Interior.
BARALE,R.; MIGLIORE,L.; CELLINI,B.; FRANCIONI, L.; GIORGELLI, F.; BARRAI, T. \& LOPRIENO, N. (1990). Genetic toxicology of airborne particulate matter using cytogenetic assays and microbial mutagenicity assays. In Genetic toxicology of complex mixtures, Plenum, 57-71.

BARALE, R.; GIORGELLI, F.; SCARPATO, R. SCAPOLI, C. LOPRIENO, N. \& BARRAI, I. (1991b). Correlation between mutagenicity of airborne particles and air pollution parameters in eleven italian towns. Int. J. Env. Healt Res., 1, 37-53.

BARALE, R.; GIROMINI, L.; DEL RY, S.; BARNINI, B.; BULLERY, M.; BARRAI, I.; VALERIO, F.; PALA, M. \& HE, J. (1994). Chemical and mutagenic patterns of airborne particulate matter collected in 17 Italian towns. Env. Healt Persp. Suppl., 102 (4), 67-73.

BARALE, R.; GIROMINI, L.; SCAPOLI, C.; LOPRIENO, M.; PALA, M.; VALERIO, F. \& BARRAI, I. (1991a). Correlations between 15 polycyclic aromatic hydrocarbons $(P A H)$ and the mutagenicity of the total $P A H$ fraction in ambient air particles in La Spezia (Italy). Mutation Res., 249, 227-241.

BARBER, S. (1993). Control \& Remediation of contaminated land: A review of available options, current best practice and comparative costs. Contaminated Land Seminar, Camborne School of Mines, Univ. of Exeter.

BARDOS, P. (1993). Process constraints on innovative soil treatment technologies. Land Contamination \& Reclamation, 1 , $37-45$.

BEALE, J.G.; (1980). The Manager and the Environment. Pergamon.

BENAYAS, J. (1990). Paisajey Educación Ambiental. Evaluación de cambios de actitudes hacia el entorno. Tesis doctoral, Univ. Autónoma de Madrid.

BHATTACHARYA, J.; MUKHOPADHYAY, S.K. \& SAMIR, K.P.; (1994). Pricing -A means to rectify environmental damage in mineral industry. In Paithankar, A.G. (ed.), The Impact of Mining on the Environment: Problems and Solutions, Balkema and Oxford \& IBH, 417-422.

BHOLE, A.G. (1994). Acid Mine Drainage and its Treatment. 
In Paithankar, A.G. (ed.), The Impact of Mining on the Environment: Problems and Solutions, Balkema and Oxford \& IBH, 131-141.

BRADSHAW, A.D. \& CHADWICK, M.J. (1980). The Restoration of Land: the Ecology and Reclamation of Derelicted and Degraded Land. Blackwell.

BROOKES, A. (1992). Recovery and restoration of some engineered british river channels. In Boom, P.J.; Calow, P. \& Petts, River Conservation and Management, J. Wiley \& Sons, 337-352.

BURROWS, P. (1980). The Economic Theory of Pollution Control. M.I.T. Press.

COMMON, M. (1988). Environmental and ResourceEconomics. Longman, Londres.

COPPIN, N.J. \& BRADSHAW, A.D. (1982). Quarry reclamation. Mining Journal Books.

DALY, H. (1990). Economics, Ecology, Ethics: Essays towards a Steady-State Economy. Freeman.

DAVIES, B.E. (1983). Heavy metals contamination from base metal mining and smelting: Implications for man and his environment. In I. Thornton, Applied Environmental Geochemistry, Academic Press.

DEAN, A.; STONE, I.; ROBERTS, J. \& MEREFIELD, J.R. (1992). Depositional Gauge Comparative Analysis. Report ERC 92/28, Univ. of Exeter.

DEWO(1989). The Reclamation of Mineral Workings. Minerals Planning Guidance. Dept. of the Environment, Welsh Office.

DOWN, C.G. \& STOKES, J. (1977). Environmental Impact of Mining. Applied Sci. Publ.

FARACO MUNUERA, F. (1986). Portmán. Uno de los mayores atentados a la ecología marina del Mediterráneo. Informes, Greenpeace, no publicado.

FILHO, E.R. (1994). The impact of mining on the environment: necessary evil or merely a calamity? In Paithankar, A.G. (ed.), The Impact of Mining on the Environment: Problems and Solutions, Balkema and Oxford \& IBH, 429-434.

FLOR, E. y MARTÍNEZ, P. (1989). Memoria del Parque del Macizo de Peña Cabarga. No publicado.

FUENTES BODELÓN, F. (1994. Marcojurídico e institucional dela normativa ambiental aplicada a las actividades extractivas en la Comunidad Europea, nacional y CC.AA. I Congreso nacional de restauración de espacios afectados por actividades extractivas. Libro de Ponencias. Grupo de Ingeniería Medioambiental, 222-241.

GIL DIAZ, J.L.; FLOR PÉREZ, E'; MARTÍNEZ EEDRÚN, P. y RODRÍGUEZ AVELLÓ, J. (1990). Parque dela Naturalezade Cabárceno (Cantabria) .Un ejemplo de restauración de un espacio afectado por explotaciones mineras a cielo abierto. IV Reunión Nacional de Geología Ambiental y Ordenación del Territorio, com., 271-278.

GIMA (1994). I Congreso nacional de restauración de espacios afectados por actividades extractivas. Libro de Ponencias. Grupo de Ingeniería Medioambiental

GÓMEZ DE COS, M.F.; PEDRAJO PÉREZ, M.A. y GONZÁLEZCUBILLOS, A.I. (1980). Explotaciónytratamiento de tierras mineralizadas en el Karst de Peña Cabarga. No publicado.

GONZÁLEZBERNALDEZ, F. (1985). Invitación a la Ecología Humana. La adaptación afectiva al entorno. Tecnos.

GONZÁLEZ GÓMEZ, I. y BAÑOS PAEZ, P. (1987) Problemática de Portmán. Anse.

GREEN, J. ed. (1992). A userguidetopit and Quarry Reclamation in Alberta. Alberta Land Conservation and Reclamation Council

GRIFFITH, J.J. (1980). Recuperaçăo Conservacionista de Superficies Mineradas. Bol. Technico 2, Sociedade de investigaçoès Florestais Viscosa, MG, Univ. Federal de Viscosa

HMIP; (1991). Environmental effects of Surface Mineral Workings. DoE, HMIP Research Report, Roy Waller Associates, HMSO.

IBAMA (1990). Manual de recuperaçăo de Areas Degradadas pela Mineraçáo. Instituto Brasileiro do Meio Ambiente Recursos Naturais Renováveis.

IBRAM(1992). Mineraçăo e Meio Ambiente, Instituto Brasileiro de Mineraçao.

JA (1993a). Plan de mejora ambiental de la cuenca del Tinto y Odiel. Junta de Andalucía, Consejería de Medio Ambiente, DG de Protección Ambiental. Inédito.

JA (1993b). Resumen de actividades de integración paisajística en minas abandonadas de la cuenca del Odiel. Junta de Andalucía, Consejería de Medio Ambiente, DG de Protección Ambiental. Inédito.

JARAMILLO GÓMEZ, A. (1994). La fauna en la restauración. Medidas correctoras. I Congreso nacional de restauración de espacios afectados por actividades extractivas. Libro de Ponencias. Grupo de Ingeniería Medioambiental, 262-267.

JASPER, D.A.; ROBSON, A.D. \& ABBOT, L.K. (1988) Revegetation in an Iron-ore mine. Nutrient requeriments for plants growth and the potential role of vesicular-arbuscular (VA) mycorrhizal fungi. Aust. J. Soil Res., 26, 497-507.

KELLY, M.; ALLISON, W.J. GARMAN, A.R. \& SYMON, C.J. (1988). Mining and the freshwater environment. Elsevier.

KING, A.M.;FRYER, G.A. \& WRIGHT,H.;(1991). Assessment of dust emissions from surface coal mining operations. Final Report, Commission of the Eropean Communities, Project $\mathbf{n}^{\circ}$ 7263-02/007/08. 
KROPP, B.R. \& LANGLOIS, G.G. (1990). Ectomycorrhizae in reforestation. Can. J. For. Res., 20, 438-450.

LARSON, B.A. \& BROMLEY, D.W. (1990). Property Rights, Externalities, and Resource Degradation: Locating the Tragedy. Jour. of Environmental Economics and Management, 8, 227237.

LEPPERT, D. (1989). Heavy metal sorption with clinoptiolite zeolite: Soil and water treatment alternatives. Soc. Min. Eng., reprint $n^{\circ} 89$.

LIBRANDO, V. (1994). Multivariate Analysis of Polycyclic Aromatic Compounds inSuspended Particulate, Polycyclic Arom. Compounds, 5, 175-183.

LLANO, A. (1988) La nueva sensibilidad. Espasa.

LORENZO AGUDO, J. Y APODACA CARRO, J.G. (1994). Restauración de terrenos en minería de carbón a cielo abierto. Descubierta de Encasur en Puertollano (Ciudad Real). En GIMA, I Congreso nacional de restauración deespacios afectados por actividades extractivas. Libro de Ponencias, 126-139.

MACEDO, a.b. (1994). Reclamation of mined lands in Brazil. In Paithankar, A.G. (ed.), The Impact of Mining on the Environment: Problems and Solutions, Balkema and Oxford \& IBH, 423-428.

MAISTERRA ARROYO, N. (1990). Proyecto de recuperación paisajística de la Cantera de Cuchía. Informe inédito.

MAITI, S.K. \& BANERJEE, S.P. (1994). Revegetation of coal mine spoils by application of domestic raw sewage. In Paithankar, A.G. (ed.), The Impact of Mining on the Environment: Problems and Solutions, Balkema and Oxford \& IBH, 369-379.

MAMANO MARTÍNEZ, J.J. (1993). Recuperación paisajística de la cantera de Cuchía. Solvay-Mitosa, Informe inédito.

MANDAVGANE, S.K.; DHARMADHIKARI, D.M. \& DARA, S.S. (1994). Reclamation of Heavy Metal Irons from Mine Water by Ferritizacion. In Paithankar, A.G. (ed.), The Impact of Mining on the Environment: Problems and Solutions, Balkema and Oxford \& IBH, 103-112.

MARRODAN ARECHAVALETA, J.I. y ABRIL GÓMEZ, H. (1993). Minería a cielo abierto y medio ambiente en Andorra (Teruel). Tecnoambiente, $\mathrm{n}^{\circ} 28,45-48$.

MARTINEZ-ALLIER, J. (1989). Ecologic and Economic Valuation. Dept. Economía e Historia Económica. Discursion paper WP 128.89.

MARX, D.H. (1991). The practical significance of ectomycorrhizae in forest establishment. In Ecophysiology of ectomycorrhizae of forest trees. Proc. Marcus Wallemberg Foundation $\mathrm{n}^{\circ} 7,54-90$.

MEREFIELD, J.R. REES, G.; STONE, I.; ROBERTS, J.; PARKES, C. \& JONES, J.; (1992). Mineralogical characterisation of atmosferic dust within and adjcent to opencoast coal sites in South Wales. Proc. Ussher Soc., 8, 67-69.
MEREFIELD, J.R.; STONE, I.; REES, G.; ROBERTS, J.; DEAN, A. \& JONES, J. (1994). Mineralogy and provenance of airborne dust in opencast coal mining areas of South Wales. Proc. Ussher Soc., 8, 313-316.

MEREFIELD, J.R.; STONE, I.; ROBERTS, J.; DEAN, A. \& JONES, J. (1995a). Monitoring airborne dust from quarrying and surface mining operations. Trans. Instn. Min. \& Metall. (sect. A: Min. industry), 104, A78-78.

MEREFIELD, J.R.; STONE, I.; JARMAN, P.; REES, G.; ROBERTS, J.; JONES, J. \& DEAN, A. (1995b). Environmental dust analysis in opencast mining areas. In M.K.G. Whately \& D.A. SPEARS, European Coal Geology, Geological Soc. Special Publ. $n^{\circ} 82,181-188$

MILLS, E.S. (1978). The economics of environmental quality. Norton.

MITCHELL, P.B. (1990). Reclaiming derelict metalliferous minig land - with particular reference to Cornwall, UK. Land and Minerals Surveying, 8 (1), 7-17.

MITCHELL, P.B. \& ATKINSON, K. (1991). The novel use of ion exange materials as an aid to reclaiming derelict mining lands. Minerals Engineering 4 (7-11), 1091-1113.

MONTORO ROMERO, C. (ed.) (1990). Medio Ambiente y crecimiento económico. Revista del Instituto de Estudios Económicos, núm. 2/1990.

MURRAY, M.Y. (1982). Waste treatment and utilization theory and practice of waste management, vol. 2, Pergamon.

NICOLAIESEN, J. (coord.) (1991). Economics and the Environment: A Survey of Issues and Policy Options. OECD Economic Studies, 16, 8-43.

ORIA DE RUEDA, J.A. (1987). Ecología del género "Suillus" en Almería y aplicación de las micorrizas de zonas áridas en repoblaciones forestales. Métodos de inoculación. FUCOVASA, E.T.S.I. de Montes, Univ. Politécnica de Madrid.

PAITHANKAR, A.G. (ed.) (1994). The impact of mining on the environment: Problems and solutions, Balkema and Oxford \& IBH.

PALACIOSUAREZ, J. (1991). El parque públicode La Arboleda (Vizcaya). Tecnoambiente, 2, 59-72.

PEARCE, D. (1976). Environmental Economics. Longman

PEARCE, D. \& TURNER, R.K. (1990). Economics of Natural Resources and the Enviroment. The John Hopkins Univ. Press.

PIRAZZI, R. \& GREGORIO, A. (1987). Acrescimento di conifere micorrizate con spezie diverse de "Tuber" spp. Mic. Ital., 16, 3, 49-62.

RAMOS, A. (1987). Ecologismo y movimientos ecologistas. En GER, XXV, 590-600, Rialp. 
RAMOS, A. (1993). ¿Por qué la conservación dela Naturaleza? Discurso leído en el acto de su recepción en la Real Academia de Ciencias Exactas, Físicas y Naturales. Fundación Conde del Valle Salazar, Madrid.

RILEY, S.J.; WAGGITT, P.W: \& QUADE C. Mc (1993). Proceedings of the Symposium on the Management and Rehabilitation of Waste Rock Dumps. Australian Governement Publ. Serv.

RMC; (1987). A practical guide to restoration. RMC group.

RODRÍGUEZ BARREAL, J.A. (1989). Importancia de la micorrización artificial de diversas especies forestales españolas. Bol. San. Veg. Plagas, 15.

RODRÍGUEZ BARREAL, J.A. (1994). Revegetación con plantas micorrizadas en canteras y escombreras. I Congreso nacional de restauración de espacios afectados por actividades extractivas. Libro de Ponencias. Grupo de Ingeniería Medioambiental, 241-262.

ROHOU, P.; BARBANSON, L.; LANDIN SAENZ, M. et PERTHUISOT, V. (1980). Un example de karst fossile dans l'Urgonien cantabrique: la mine de fer d'Orconera (Province de Santander, Espagne). Jornadas sobre el karst en Euskadi, 301306.

RUEHLE, J.L. (1980). Growth of containerized loblolly pine with specific ectomycorrhizae after 2 years on an amended borrow pit. Reclamation Rev., 3, 95-101.

SAIZ DE OMEÑACA, J. (1987). Impactos Ambientales de la Mineria. En Ramos, A. (coord.) Diccionario de la Naturaleza ESPASA-CALPE, 517-520, Madrid.

SAIZ DE OMEÑACA, J. (1991). Dictamen sobre efectos geoambientales derivados de la instalación y uso de la central hormigonera de Puente San Miguel (Reocin, Cantabria). No publicado.

SAIZ DEOMEÑACA, J.; ANTÓNFERRER, R.; CALATAYUD PRIETO, T.; CIFUENTES VEGA, P.; ORTEGA VALCARCEL, J.; SAIZ DE OMEÑACA, G. Y SAIZ DE OMEÑACA, J.A. (1981). Utilización didáctica del Medio Ambiente (La Bahía de Santander). Instituto de Ciencias de la Educación, Universidad de Santander.

SAIZ DE OMEÑACA, J.; EREÑO, I.; ATXABAL, K. and AZURMENDI, I. (1993a). Reclamation of adverse effects at the Lezama-Leguizamonabandoned open pit mine (Bilbao, N. Spain). Environmental Geology, vol. 22, (1), 10-12.

SAIZ DE OMEÑACA, J.; EREÑO, I.; GÓMEZ, D. y SOLAR, M. (1993b). Los movimientos de masa en la ladera de Irala (Bilbao): origen, consecuencias y bases para el diseño de soluciones. Problemática Geoambiental y Desarrollo, 651-658.

SAIZ DE OMEÑACA, J.; RODRÍGUEZ BARREAL, J.A.; ZAZO, J.and SAIZ DE OMEÑACA, J.A. (1994b). Mycorrhyzation: a technique for arid climates and poor soils. Experiences in Almeria with "Lactarius deliciosus", "Suillus granulatus" and "Tuber melanosporum". In Paithankar, A.G. (ed.). The impact of mining on the environment: Problems and solutions, Balkema and Oxford \& IBH, 311-320.

SAIZ DE OMEÑACA, J.; RODRIGUEZ BARREAL, J.A.; ZAZO MUCHARAZ, J. y SAIZ DE OMEÑACA, J.A. (1994c). Micorrizas y revegetación. Experiencias con pino carrasco artificialmente micorrizado en las sierras de Filabres y Lúcar (Almeria). Papeles del Centro EIA, 129-133.

SAIZ DE OMEÑACA, J. y SAIZ DE OMEÑACA, J.A. (1993a). El medio ambiente, la adhesión a las comunidades europeas y la empresa española. Cantárida, 120, 23-25.

SAIZ DE OMEÑACA, J. y SAIZ DE OMEÑACA, J.A. (1993b).

El medio ambiente, la adhesión a las comunidades europeas y la empresa española. Cantárida, 121, 20-21.

SAIZ DE OMEÑACA, J.; SAIZ DE OMEÑACA, J.A. y SERNA, M.R. (1990). Guía para colaboración en estudios de evaluación de impacto sobre cavidades cársticas. Actas del V Congreso Nacional de Espeleología, 29-33.

SAIZ DE OMEÑACA, J.; SOLAR, M.; GÓMEZ, D.; MARCOS, J. y SAIZ DE OMEÑACA, M.G. (1992). Plan de Restauración de la Cantera Blanca y de la Cantera Gris de Montehano (Escalante, Cantabria). Concesiones mineras Dolomitica y Aumento a Dolomitica. No publicado.

SAIZDE OMEÑACA, J.; SOLAR, M.; GÓMEZ, D.; MARCOS, J.; SAIZ DE OMEÑACA, M.G. and SAIZ DE OMEÑACA, J.A. (1994a). Open cast mine reclamation in Spain: a review with particularreference tothe Montehano Mine (Cantabria, Northern Spain). In Paithankar, A.G. (ed.), The Impact of Mining on the Environment: Problems and Solutions, Balkema and Oxford \& IBH, 355-368.

SAIZ DE OMEÑACA, J.; TRUJILLANO, A.; SOLAR, M. y GÓMEZ,D. (1994d). Estudios deviabilidad técnica y ambiental de vertederos de residuos sólidos en Santander. La Pasadillay Cueto. III Congreso de la red española de ciudades saludables, Ponencias y comunicaciones, 237-245.

SCARPATO, R.; DI MARINO, F.; STRANO, A.; CURTI, A.; CAMPAGNA, R.; LOPRIENO, N.; BARRAL, I. \& BARALE, R. (1993). Two years' air mutagenicity monitoring in a northwestern rural area of Italy with an industrial plant. Mutation Res., 319, 293-301

SENDLIN, H.; YAZIAGIL, V.A. \& CARLSON, C.L. (1983). Surface Mining Environmental Monitoring and Reclamation Handbook. Elsevier.

SERRANO AGUILAR, J. (1994). Contaminación por aguas ácidas procedentes de minas. Proyecto de regeneración de los rios Tinto y Odiel. RETEMA, nº 39, 9-15.

SMITH, J.W. (1988). The beneficial use and disposal of coal ash on active mine sites in Pensilvania. In Mine Drainage and Surface Mine Reclamation, vol 2: Mine reclamation, abandoned mine lands and policy issues, Bureau of Mines, circ. IC 9184 , US Dept. of Interior, 285-293. 
SMITH, M. (1993). Dealing with contaminated land conditions. Land Contamination and Reclamation, 1 (1), 22-36

SOLAR FERNÁNDEZ, M.; GÓMEZ MARTÍNEZ, D.; SAIZ DE OMEÑACA, M.G.; MARCOS MARTÍNEZ, J. y SAIZ DE OMEÑACA, J. (1993). El plan de restauración de las canteras de Montehano (Escalante, Cantabria): la preparación del substrato para implantar vegetación. Tecnoambiente, 30, 5963.

SOLOW, R. (1974). The Economics of Resources orthe Resources of Economics. American Economic Review, 64, 1-14.

STEWART, H.T.L. HOPMAN, P. FLINN, P. \& HILMANN, T.J. (1990). Nutrient accumulation in trees and soil following irrigation with municipal effluent in Australia. Environ. Pollute, 63, 156-177.

TIETEMBERG, T.H. (1990). Economic Instruments for Environmental Regulations. Oxford Review of Economic Policy, 6, 17-33.
TOY, T.J. \& HADLEY, R.F. (1987). Geomorphology and reclamation of disturbed lands. Academic Press.

VARIOS AUTORES; (1992). Recuperação deáreas degradadas. Universidade Federal do Paraná e Fundaçao de Pesquisas Florestais do Paraná.

VARIOS AUTORES (1993). Proceedings of the Symposium on the Management and Rehabilitation of Waste Rocks Dumps. Supervising Scientist for the Alligator Rivers Region.

VILAR, J.B. EGEA BRUNO, P.M. Y FERNÁNDEZ GUTÍERREZ, J.C. (1991). La minería murciana contemporánea (1935-1985). Instituto Tecnológico Geominero de España.

VIÑAS BOSQUET, L. Y LOPEZ FERNÁNDEZ, A.J. (1994). Corrección medioambiental de impactos de origen minero en la cuenca del Odiel. Residuos, n 3, 67-71.

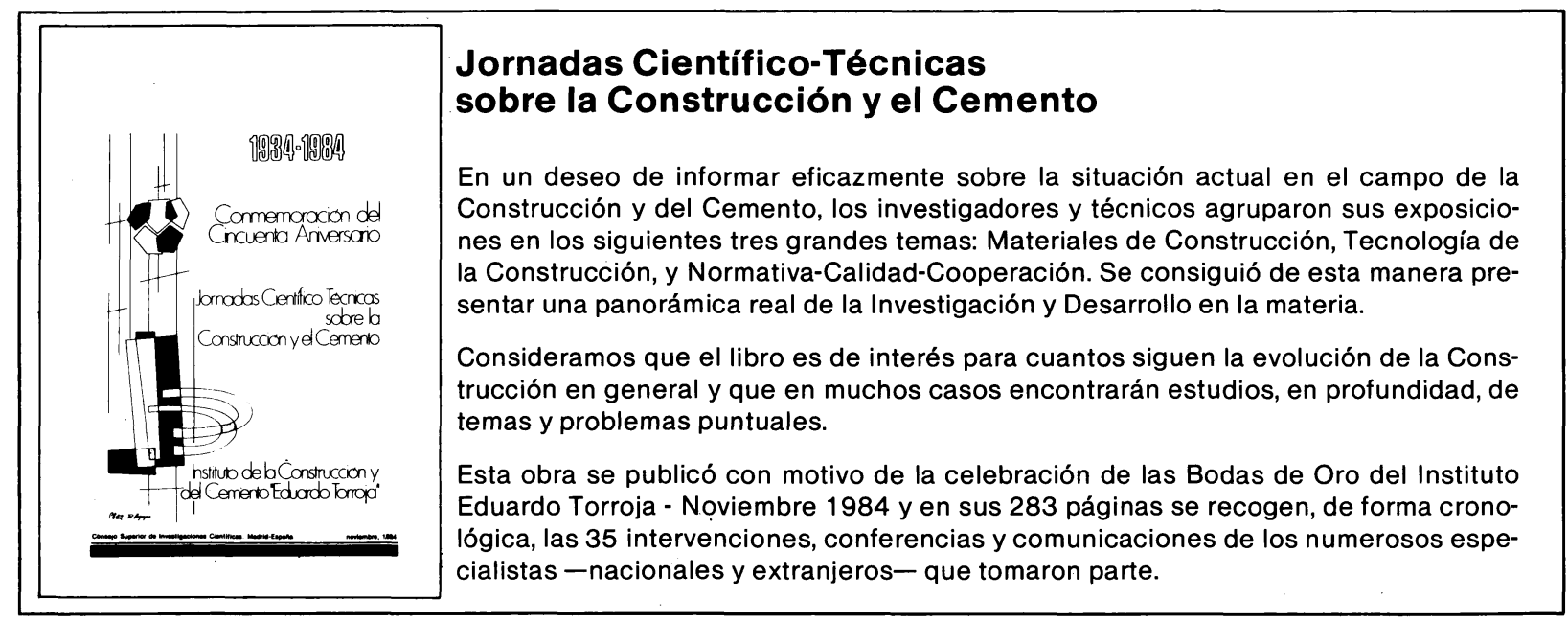

